\title{
Influence of Material Compressibility on Displacement Solution for Structural Steel Plate Applications
}

\author{
Nelli Aleksandrova \\ Centre of Exact Sciences and Engineering, University of Madeira, Funchal, 9020-105 Madeira, Portugal \\ Correspondence should be addressed to Nelli Aleksandrova; nelli@uma.pt
}

Received 4 July 2014; Accepted 12 October 2014; Published 23 October 2014

Academic Editor: Lucian Dascalescu

Copyright (C) 2014 Nelli Aleksandrova. This is an open access article distributed under the Creative Commons Attribution License, which permits unrestricted use, distribution, and reproduction in any medium, provided the original work is properly cited.

\begin{abstract}
Displacement field calculations are necessary for many structural steel engineering problems such as cold expansion of holes, embedment of bolts and rivets, and installation and maintenance of external devices. To this end, rigorous closed form analytical displacement solution is obtained for structural steel open-hole plates with in-plane loading. The material of the model is considered to be elastic perfectly plastic obeying the von Mises yield criterion with its associated flow rule. On the basis of this solution, two simplified engineering formulae are proposed and carefully discussed for practical engineering purposes. Graphical representations of results show validity of each formula as compared with rigorous solution and other studies.
\end{abstract}

\section{Introduction}

Displacement field calculation around stress concentrators in steel structural members is on-going engineering task which is necessary for a number of reasons. It provides the basis for specific industrial codes and regulations such as AISC or Eurocodes, makes substantial part of commercial software based on finite element method (FEM), and proves validity of initial stress solutions. In a large number of cases, structural elements utilized in civil, mechanical, and aerospace engineering require major repair or are out of service due to ductile failures originating at holes. To this end, two widely applied technological processes are of great importance, namely, cold expansion of holes to improve fatigue life of structural members [1] and embedment of bolts to achieve the optimum bearing area within the available hole [2]. For cold expansion process, the plate may reach purely plastic state upon loading. In contrast, for bolt connections, the plate surrounding the fastener in a global structural environment should be mostly in elastic state; that is, the plastic zone is inferior to elastic one. For mild and high strength steels, this difference in structural expectation from the technological processes may influence the methodology of displacement evaluation based on the same geometrical model of a plate with central hole subjected to internal pressure. Such a model is commonly used both in analytical and numerical calculations dealing with either cold expansion process (with its applications) [1,3-7] or specifically with bolt connections $[2,8-10]$. In spite of the fact that numerical analyses form a powerful tool in complex realistic engineering situations, analytical methods continue to occupy important role in preliminary engineering design due to their rigorous mathematical formulations and clear straightforward answers. For a plate with a hole, numerous analytical studies have been performed on various material models (rigid plastic, elastic perfectly plastic, and elastic-plastic strain hardening) and yield criteria (mostly Tresca yield criterion with its associated flow rule and von Mises yield criterion combined with deformation theory of plasticity). Predominately, the elastic-plastic and residual stress analyses have been conducted. Concerning strain analysis (or assumptions made about it) and related displacement field calculation, it is common for the reason of mathematical simplicity to consider material as incompressible in elastic zone [3] or make specific assumptions about the Poisson's coefficient leading to a variable Poisson ratio $[4,5,7]$. However, up to now, there is no study available which deduces, on rigorous basis, discusses, and justifies a formula for displacements and its specific usage for steel structural members. So the objective of the present research is to develop rigorous and approximate displacement solutions and discuss their validity taking into account the most important characteristics of the 
material-elastic modulus, yield stress, and Poisson's ratiowhich influence steel structural members. Since experimental results show that the strength of most metal materials is better described by the Mises yield condition [11], the model is based on this criterion with its associated flow rule and elastic perfectly plastic material behavior. Such material model permits getting uncoupled stress solution and considering separately strain-displacement fields. No restrictions or additional assumptions are applied except for the plane stress state and small strain theory. It is worth noting that the common use of deformation theory of plasticity, especially with additional assumptions of elastic incompressibility and neglect of the elastic strains in the plastic zone (which permits the derivation of analytical formulae for stresses and simplifies substantially the analysis), is not completely justified. So the general formulation of the problem used in the current research allows the total deformation in the plastic zone to be considered as the sum of elastic and plastic parts taking into account compressibility of the material.

\section{Statement of the Problem and Uncoupled Stress Solution}

In cylindrical coordinate system $r \theta z$ with nonzero radial, $\widehat{\sigma}_{r r}$, and tangential, $\widehat{\sigma}_{\theta \theta}$, stress tensor components, consider a thin annular plate of inner radius $a$ and outer radius $b$ subjected to gradually increasing radial pressure $\widehat{p}$ around its inner edge (Figure 1).

The following boundary conditions in stress should be satisfied:

$$
\widehat{\sigma}_{r r}=-\widehat{p} \quad \text { at } r=a, \quad \widehat{\sigma}_{r r}=0 \quad \text { at } r=b .
$$

For sufficiently small values of external loadings $\widehat{p}$, the whole plate is entirely elastic. The elastic load-carrying capacity (when the plate starts yielding) corresponds to

$$
\frac{\widehat{p}}{Y}=\frac{\left(1-(a / b)^{2}\right)}{\sqrt{3+(a / b)^{2}}}
$$

where $Y$ is the yield stress of the material in tension test. For higher loads, the plate in general consists of two zonesinner plastic and outer elastic-divided by the elastic/plastic boundary, $c$. In elastic zone, the material obeys the Hooke's law with the relevant stress-strain equations:

$$
\widehat{\varepsilon}_{r r}=\frac{\partial \widehat{u}}{\partial r}=\frac{\left(\widehat{\sigma}_{r r}-\nu \widehat{\sigma}_{\theta \theta}\right)}{E} ; \quad \widehat{\varepsilon}_{\theta \theta}=\frac{\widehat{u}}{r}=\frac{\left(\widehat{\sigma}_{\theta \theta}-\nu \widehat{\sigma}_{r r}\right)}{E},
$$

where $\widehat{\varepsilon}_{r r}$ and $\widehat{\varepsilon}_{\theta \theta}$ are the radial and tangential strains, respectively; $\widehat{u}$ is the radial displacement; $E$ is the Young modulus; and $v$ is the Poisson coefficient. In the plastic zone, the von Mises yield criterion is adopted. For plane stress state and absence of in-plane shear stresses, it is simplified to

$$
\widehat{\sigma}_{r r}^{2}+\widehat{\sigma}_{\theta \theta}^{2}-\widehat{\sigma}_{r r} \widehat{\sigma}_{\theta \theta}=Y^{2}
$$

and is automatically satisfied by the following parametric substitutions:

$$
\frac{\widehat{\sigma}_{r r}}{Y}=\left(\frac{2}{\sqrt{3}}\right) \cos \varphi, \quad \frac{\widehat{\sigma}_{\theta \theta}}{Y}=\left(\frac{2}{\sqrt{3}}\right) \cos \left(\varphi-\frac{\pi}{3}\right),
$$

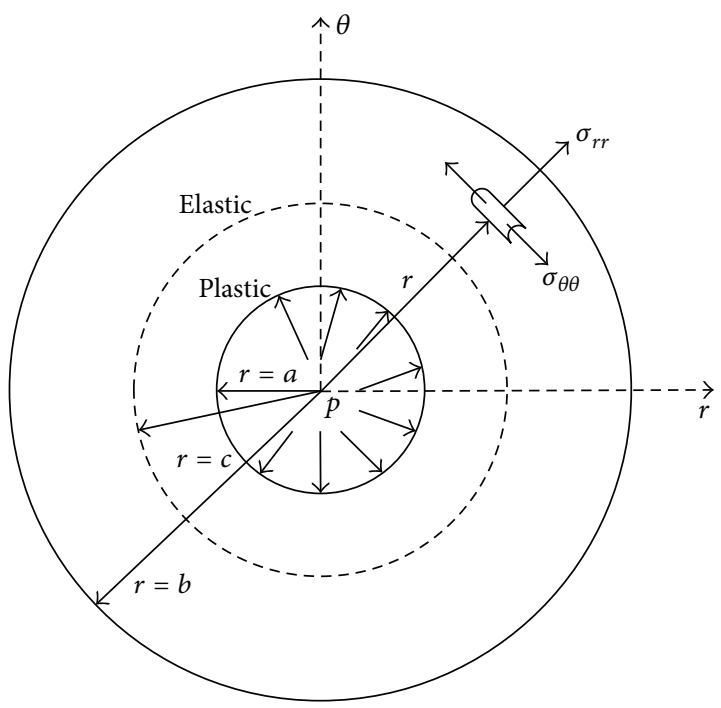

FIGURE 1: Geometrical model of a circular plate under internal pressure in cylindrical coordinate system.

where $\varphi$ is an auxiliary variable. In fact, (5) describes stress distributions in plastic zone. Due to the circular symmetry of the problem, $\widehat{\sigma}_{r r}=\widehat{\sigma}_{r r}(r), \widehat{\sigma}_{\theta \theta}=\widehat{\sigma}_{\theta \theta}(r)$, and plane stress assumption, $\widehat{\sigma}_{z z}=0$, there is only one nontrivial equilibrium equation:

$$
\frac{d \widehat{\sigma}_{r r}}{d r}+\frac{\left(\widehat{\sigma}_{r r}-\widehat{\sigma}_{\theta \theta}\right)}{r}=0
$$

which is valid in both elastic and plastic zones of the plate.

To conduct further analysis, the dimensionless parameters may be introduced $\sigma_{r r}=\widehat{\sigma}_{r r} / Y, \sigma_{\theta \theta}=\widehat{\sigma}_{\theta \theta} / Y, p=\widehat{p} / Y$, $\beta=r / b, \alpha=a / b, \gamma=c / b, \varepsilon_{r r}=\widehat{\varepsilon}_{r r} E / Y, \varepsilon_{\theta \theta}=\widehat{\varepsilon}_{\theta \theta} E / Y$, and $u=\widehat{u} E /(Y b)$. In the inner plastic zone, the solution of equilibrium equation (6) in parametric form leads to an analytical expression defining the relation between radial coordinate $\beta$ and variable $\varphi$ :

$$
\frac{\beta^{2}}{\alpha^{2}}=\frac{\sin \left(\varphi_{\alpha}-\pi / 6\right) \exp \left[\sqrt{3}\left(\varphi_{\alpha}-\varphi\right)\right]}{\sin (\varphi-\pi / 6)},
$$

where $\varphi_{a}$ is the value of $\varphi$ at $\beta=\alpha$ and is defined from the boundary conditions as

$$
\varphi_{a}=\arccos \left(-\frac{\sqrt{3} p}{2}\right) .
$$

The radius of the elastic-plastic boundary is determined by the simple formula

$$
\frac{1}{\gamma^{2}}=\frac{\operatorname{tg}\left(\varphi_{\gamma}-\pi / 6\right)}{\sqrt{3}},
$$

where $\varphi_{\gamma}$ is the value of $\varphi$ at the elastic-plastic boundary $\gamma$ which is expressed through the $\varphi_{a}$ (8) and a given geometry of the hole $\alpha$, using (7) as follows:

$$
\frac{1}{\alpha^{2}}=\frac{1}{\sqrt{3}} \exp \left[\sqrt{3}\left(\varphi_{\alpha}-\varphi_{\gamma}\right)\right] \frac{\sin \left(\varphi_{\alpha}-\pi / 6\right)}{\cos \left(\varphi_{\gamma}-\pi / 6\right)} .
$$


In the outer elastic zone, taking into account the condition of continuity of stresses at the elastic-plastic boundary, the stress-displacement solution may be also analytically defined:

$$
\begin{aligned}
\sigma_{r r}^{E} & =\left(1-\frac{1}{\beta^{2}}\right) \cos \left(\varphi_{\gamma}-\frac{\pi}{6}\right), \\
\sigma_{\theta \theta}^{E} & =\left(1+\frac{1}{\beta^{2}}\right) \cos \left(\varphi_{\gamma}-\frac{\pi}{6}\right) \\
u^{E} & =\left[(1-\nu) \beta+\frac{(1+\nu)}{\beta}\right] \cos \left(\varphi_{\gamma}-\frac{\pi}{6}\right) .
\end{aligned}
$$

\section{Kinematic Analysis: Rigorous Displacement Solution}

In general procedure (which is mathematically and physically rigorous) exploited in the present research, the total strain in the inner plastic zone is assumed to be the sum of elastic and plastic portions. The elastic portion is obtained from Hooke's law (3) and stress distributions (5):

$$
\begin{aligned}
\varepsilon_{r r}^{e} & =\frac{[(2-\nu) \cos \varphi-\sqrt{3} \nu \sin \varphi]}{\sqrt{3}} \\
\varepsilon_{\theta \theta}^{e} & =\frac{[(1-2 \nu) \cos \varphi+\sqrt{3} \nu \sin \varphi]}{\sqrt{3}} .
\end{aligned}
$$

The plastic portion is related to the associated (with the Mises yield criterion (4)) flow rule:

$$
\frac{\dot{\varepsilon}_{r r}^{p}}{\dot{\varepsilon}_{\theta \theta}^{p}}=\frac{s_{r r}}{s_{\theta \theta}},
$$

where $\dot{\varepsilon}_{r r}^{p}$ and $\dot{\varepsilon}_{\theta \theta}^{p}$ are the radial and circumferential plastic portions of strain rate components, respectively, and $s_{r r}$ and $s_{\theta \theta}$ are the radial and circumferential deviatoric components of stress tensor, respectively. At small strains, $\dot{\varepsilon}_{r r}^{p}=\partial \varepsilon_{r r}^{p} / \partial t$ and $\dot{\varepsilon}_{\theta \theta}^{p}=\partial \varepsilon_{\theta \theta}^{p} / \partial t$, where $\varepsilon_{r r}^{p}$ and $\varepsilon_{\theta \theta}^{p}$ are the radial and circumferential plastic portions of strains, respectively, and $t$ is a time factor. It follows from (5), (7), and (8) that the deviatoric components of stresses are independent of time. Therefore, (14) may be immediately integrated to give

$$
\varepsilon_{r r}^{p}=\frac{\varepsilon_{\theta \theta}^{p}(\sqrt{3} \cos \varphi-\sin \varphi)}{(2 \sin \varphi)}
$$

Due to the general statement of the problem, in the plastic zone $\varepsilon_{r r}=\varepsilon_{r r}^{e}+\varepsilon_{r r}^{p}=\partial u / \partial r$ and $\varepsilon_{\theta \theta}=\varepsilon_{\theta \theta}^{e}+\varepsilon_{\theta \theta}^{p}=u / r$. Substituting (13) and (15) into these equalities gives

$$
\begin{aligned}
\frac{\partial u}{\partial \beta} & =\frac{1}{\sqrt{3}}[(2-\nu) \cos \varphi-\sqrt{3} \nu \sin \varphi]+\varepsilon_{\theta \theta}^{p} \frac{\sqrt{3} \cos \varphi-\sin \varphi}{2 \sin \varphi} \\
\frac{u}{\beta} & =\frac{1}{\sqrt{3}}[(1-2 \nu) \cos \varphi+\sqrt{3} \sin \varphi]+\varepsilon_{\theta \theta}^{p} .
\end{aligned}
$$

Eliminating of $\varepsilon_{\theta \theta}^{p}$ results in differential equation for radial displacement in plastic zone:

$$
\frac{\partial u}{\partial \beta}-\frac{\sqrt{3} \cos \varphi-\sin \varphi}{2 \sin \varphi} \frac{u}{\beta}=\frac{1-2 \nu}{2 \sqrt{3} \sin \varphi}(\sin 2 \varphi-\sqrt{3} \cos 2 \varphi)
$$

which may be rewritten using variable $\varphi$ (to be consistent with the analysis presented) as independent variable (instead of $\beta$ ):

$$
\begin{aligned}
\frac{d u}{d \varphi}- & \frac{\sin (\varphi-\pi / 3)}{\sin (\varphi-\pi / 6)} u \\
= & -\frac{(1-2 \nu) \alpha}{2 \sqrt{3}} \frac{(\sin 2 \varphi-\sqrt{3} \cos 2 \varphi)}{[\sin (\varphi-\pi / 6)]^{3 / 2}} \\
& \times \exp \left[\frac{\sqrt{3}}{2}\left(\varphi_{\alpha}-\varphi\right)\right] \sqrt{\sin \left(\varphi_{\alpha}-\frac{\pi}{6}\right)} .
\end{aligned}
$$

For rigorous calculations of radial displacements, (18) represents the most general form which takes into account material compressibility in the plastic zone. To finalize the procedure, analytical solution of this equation can be obtained. It requires some mathematical efforts but, once done, it helps to analyze in detailed manner various simplifications for engineering usage. So, analytically, the general formula for the radial displacement in plastic zone has the form

$$
\begin{aligned}
u(\varphi)= & \frac{\exp [(\sqrt{3} / 2)(\varphi-\pi / 6)]}{\sqrt{\sin (\varphi-\pi / 6)}} \\
& \times\left(C+\int_{\varphi_{\gamma}}^{\varphi} \frac{\sqrt{\sin (\xi-\pi / 6)}}{\exp [(\sqrt{3} / 2)(\xi-\pi / 6)]} \Phi_{1}(\xi) d \xi\right),
\end{aligned}
$$

where $\Phi_{1}$ is the right-hand side of (18) and $C$ is a constant. Since the radial displacement $u$ is a continuous function across the elastic-plastic boundary, the constant $C$ in (19) is determined from the continuity condition for displacements:

$$
u\left(\varphi_{\gamma}\right)=u_{\gamma}
$$

where $u_{\gamma}$ is the value of $u$ at the elastic-plastic boundary, is obtained from (12) by putting $\beta=\gamma$, and, using (9), becomes

$$
u_{\gamma}=\gamma\left[\left(1-\nu^{E}\right) \cos \left(\varphi_{\gamma}-\frac{\pi}{6}\right)+\frac{\left(1+\nu^{E}\right) \sin \left(\varphi_{\gamma}-\pi / 6\right)}{\sqrt{3}}\right] \text {, }
$$

where upper index " $E$ " is attributed to Poisson's ratio in the outer elastic zone (just to track it more explicitly). After combining (19)-(21) one gets mathematically rigorous and 
physically consistent expression for radial displacement in the plastic zone:

$$
\begin{aligned}
u= & \gamma\left[\left(1-\nu^{E}\right) \cos \left(\varphi_{\gamma}-\frac{\pi}{6}\right)+\frac{1}{\sqrt{3}}\left(1+\nu^{E}\right) \sin \left(\varphi_{\gamma}-\frac{\pi}{6}\right)\right] \\
& \times \exp \left[\frac{\sqrt{3}}{2}\left(\varphi-\varphi_{\gamma}\right)\right] \sqrt{\frac{\sin \left(\varphi_{\gamma}-\pi / 6\right)}{\sin (\varphi-\pi / 6)}} \\
& +\frac{1-2 \nu}{2 \sqrt{3}} \alpha \sqrt{\frac{\sin \left(\varphi_{\alpha}-\pi / 6\right)}{\sin (\varphi-\pi / 6)}} \exp \left[\frac{\sqrt{3}}{2}\left(\varphi_{\alpha}+\varphi\right)\right] \\
& \times \int_{\varphi_{\gamma}}^{\varphi} \Phi_{2}(\xi) d \xi
\end{aligned}
$$

where $\Phi_{2}(\xi)=-((\sin 2 \xi-\sqrt{3} \cos 2 \xi) / \sin (\xi-\pi / 6))$ $\times \exp [-\sqrt{3} \xi]$.

The total displacement along the radius is the sum of (12) where Poisson's ratio should be read as $v^{E}$ and (22). In (22), it should be noticed that, for physically consistent solution, Poisson's ratios related to elasticity in both inner plastic, $v$, and outer elastic, $\nu^{E}$, zones should be identical.

\section{Engineering Analysis of Displacement Field}

As it follows from the procedure of deducing (17), the righthand side of this equation corresponds to the contribution of elastic portion of strains in plastic zone (13) to general kinematic analysis. Neglecting elastic strains mathematically would be equivalent to the statement of elastically incompressible material in the plastic zone (which follows directly from (17) by putting $v=1 / 2$ ). In this case, the governing differential equation (18) and its analytical solution (19) are drastically simplified:

$$
\begin{gathered}
\frac{d u}{d \varphi}-\frac{\sin (\varphi-\pi / 3)}{\sin (\varphi-\pi / 6)} u=0, \\
u(\varphi)=\frac{C \exp [(\sqrt{3} / 2)(\varphi-\pi / 6)]}{\sqrt{\sin (\varphi-\pi / 6)}} .
\end{gathered}
$$

The constant $C$ now is found form (20)-(21) where the coefficient of Poisson arises from within the outer elastic zone. So, formally (however, not physically consistent), (24) may be rewritten as

$$
\begin{aligned}
u= & \gamma\left[\left(1-\nu^{E}\right) \cos \left(\varphi_{\gamma}-\frac{\pi}{6}\right)+\frac{1}{\sqrt{3}}\left(1+\nu^{E}\right) \sin \left(\varphi_{\gamma}-\frac{\pi}{6}\right)\right] \\
& \times \exp \left[\frac{\sqrt{3}}{2}\left(\varphi-\varphi_{\gamma}\right)\right] \sqrt{\frac{\sin \left(\varphi_{\gamma}-\pi / 6\right)}{\sin (\varphi-\pi / 6)}},
\end{aligned}
$$

where $v^{E}$ stands for Poisson's ratio in the outer elastic zone (to distinguish it from $v=1 / 2$ suggested in the inner plastic zone for this particular engineering approximation).
Another simplification of general displacement solution (22) may be reached by suggesting material to be incompressible both in the outer elastic and inner plastic zones (physically consistent assumption); that is $v^{E}=1 / 2$ in (21), leading to

$$
u=\gamma \sin \varphi_{\gamma} \exp \left[\frac{\sqrt{3}}{2}\left(\varphi-\varphi_{\gamma}\right)\right] \sqrt{\frac{\sin \left(\varphi_{\gamma}-\pi / 6\right)}{\sin (\varphi-\pi / 6)}} .
$$

In the outer elastic zone, the formula for the radial displacement is the same as in the general case, and the total displacement is the sum of solutions in the inner plastic and outer elastic zones. To exploit the range of validity of approximate engineering solutions (25) and (26) in the inner plastic zone, numerical calculations have been performed and compared with the general rigorous solution (22). Figure 2 shows displacement distributions in the inner plastic zone for a typical steel Poisson's ratio $\nu=0.3$ and medium load $p=0.6$ which brings the plate material into partial elastic/plastic state where the plastic zone is still rather small. Here and in all other graphs, solid lines correspond to the rigorous exact solution given by (22). Obviously, solid lines for $v=0.5$ reflect the same results obtained from (22) or (26). The dashed lines correspond to the first approximate solution (25) at $v=0.3$. The geometrical parameter is chosen to be $\alpha=0.5$.

The same type of displacement distributions is shown in Figure 3 with the exception of the external load $p=0.7697$ which in this case brings the plate material into fully plastic state.

It can be seen from both figures that, for $v=0.3$, the general formula (22) predicts slightly lower value of displacement than the approximate formula (25). Obviously, for $v=0.5$, which corresponds to the upper limit of radial displacements, the general solution (22) and the most simple approximate solution (26) are numerically coincident. However, any solution for $v=0.5$ is too conservative for lower loads or, in other words, when the plastic zone is not too pronounced (Figure 2). In contrast, for developed plastic zones, the simplest formula (26) might be considered for suitable engineering applications as it can be seen from Figure 3 where the dashed line for $v=0.3$ (first approximate solution (25)) coincides with the solid line for $v=0.5$. To compare more precisely the rigorous formula (22) and approximate one (25), two more figures are presented-Figure 4 (for $p=$ 0.6 ) and Figure 5 (for $p=0.7697$ ) - at radius location $\beta=0.5$ (edge of the hole where the influence of Poisson's ratio is more pronounced). These figures show distributions of radial displacements in the inner plastic zone versus Poisson's ratio.

For medium or lower loads (Figure 4) and real values of Poisson's ratios for steel structural members, the error in calculation by the approximate formula (25) (dashed lines) is insignificant and this formula can be safely used. Interesting situation takes place for higher loads (Figure 5) when two approximate solutions (25) and (26) coincide (dashed lines); however, both of these solutions might be inaccurate for lower values of Poisson's ratio and hence general formula (22) is required. To complete the analysis, one more figure is drawn-Figure 6 for $p=0.6$-which shows distributions of total radial displacements along the radius. 


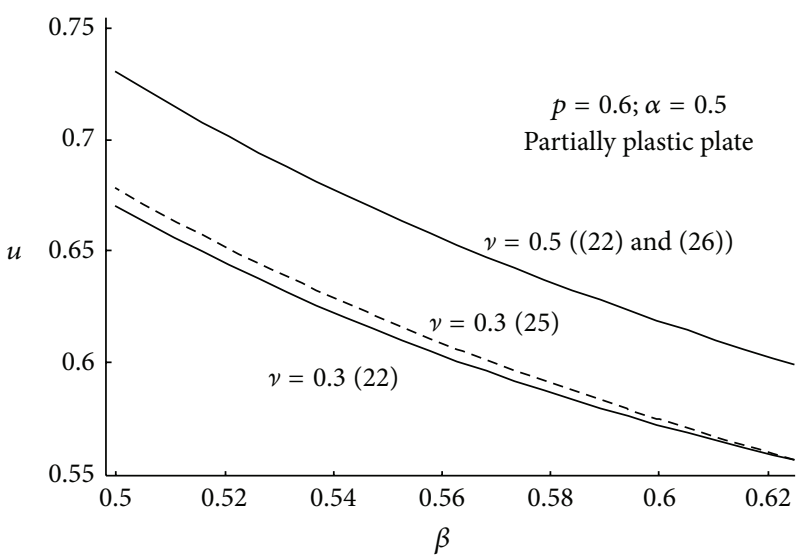

FIgURE 2: Radial displacement distributions in plastic zone for medium pressure.

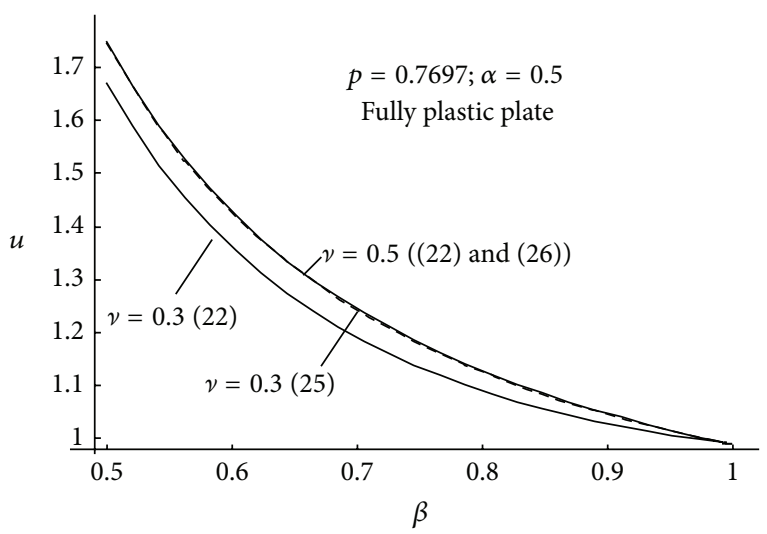

FIGURE 3: Radial displacement distributions for completely plasticized material due to high pressure.

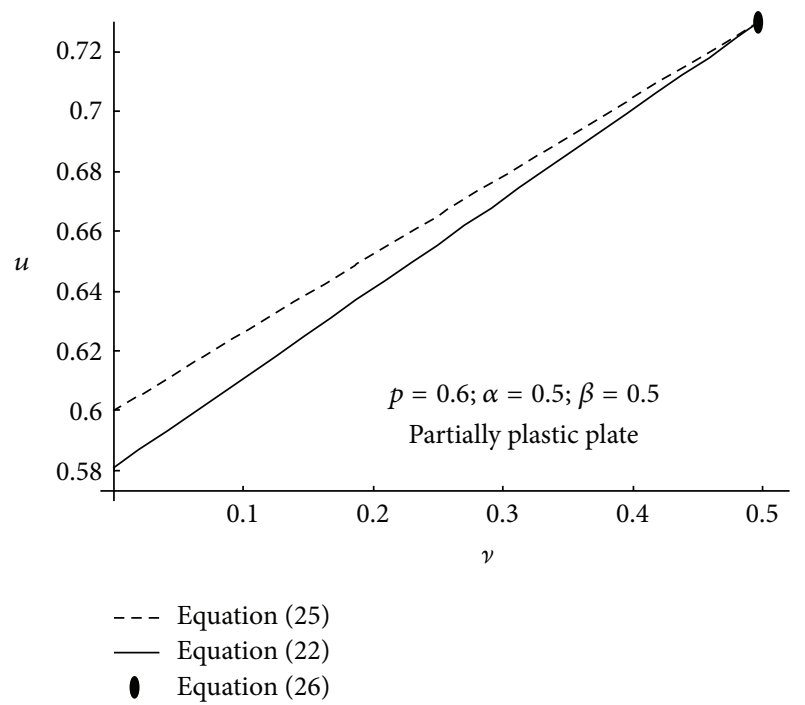

FIGURE 4: Dependence of radial displacement in plastic zone on material compressibility for medium pressure.

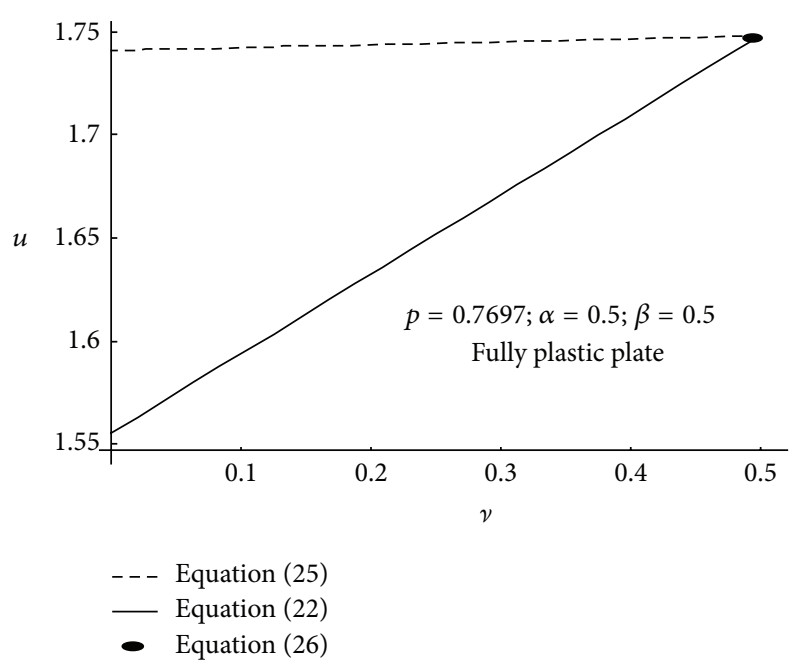

FIGURE 5: Dependence of radial displacement in completely plasticized plate on material compressibility.

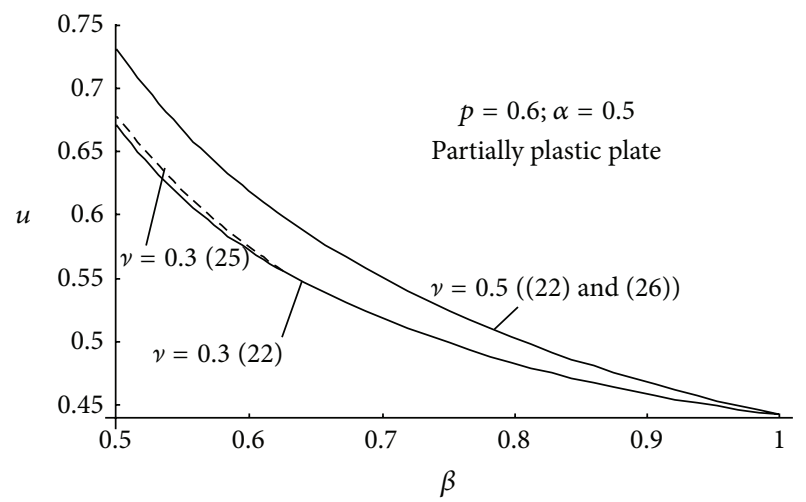

FIGURE 6: Displacement distributions versus radius for medium pressure calculated on the basis of various engineering approximations.

As it can be seen from this figure, the difference between rigorous solution (22) and first approximate solution (25) for $\nu=0.3$ is insignificant for medium to lower external loading.

\section{Discussion and Conclusions}

The analyses presented in this study are based on the rigorous formula (22) which also can be used for comparison with other works. To this end, Figure 7 shows dependence of the radial expansion $u / u_{0}$ on practical values of pressure load $p$, $u_{0}$ being the radial enlargement of the hole when the yielding point is just reached at $r=a$. Solid line corresponds to the results obtained by the present solution, and dots stand for the results retrieved from the related work [5] after proper adjustments of mechanical and geometrical parameters.

It can be seen from Figure 7 that an excellent agreement has been reached for all practical values of internal pressure. The only discrepancy is observed for higher theoretical pressure and is due to the original hardening model considered in [5] (the hardening exponent parameter $n$ in the hardening 


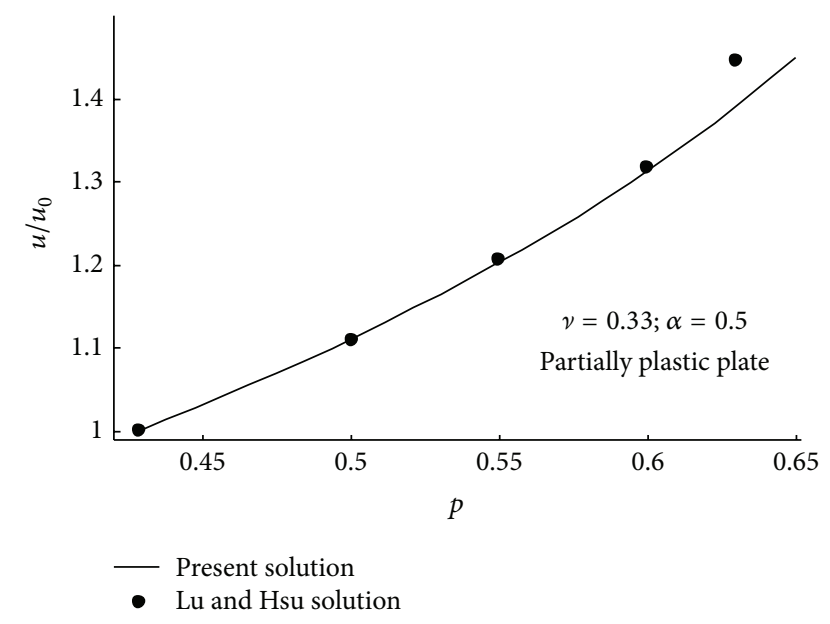

FIgURE 7: Dependence of radial expansion on practical values of pressure load.

model was chosen $n=200$ to approximate elastic perfectly plastic material). For this approximation and specific geometry $b / a=2$, three characteristic values of nondimensional pressure were considered in [5]: $p=0.5059 ; 0.7033 ; 0.7724$, and the corresponding values of elastic-plastic border radius were calculated: $c / a=1.1 ; 1.5 ; 2$, respectively. These results are coincident with those obtained in the present study except for the last $P$ value where the adjustment is required for an elastic perfectly plastic material for which the plate reaches its fully plastic state at $p=0.7697$ (i.e., the error due to material modeling being around $0.35 \%$ ). So it can be also concluded that, in general, hardening influences significantly structural behavior of fastener plates leading to higher values of internal pressure necessary to produce the same radial enlargement of the hole. It is also interesting to note that assumption of geometrically infinite plate also leads to higher values of internal pressure compared to those predicted by a sizable annular plate as compared with $[5,10]$.

Taking into account that, in real engineering applications, the stress state near the hole is rather complex, the proposed simplified solutions can be effectively used in the preliminary design stage to assist three-dimensional complete numerical modeling usually applied to most engineering structures. Based on a general analytical elastic perfectly plastic displacement analysis presented, elastic-plastic boundaries in plate structures and permanent enlargement of open holes due to in-plane loading are easily assessable. These data are required to select optional material/geometrical parameters such as yield limit, elastic modulus, Poisson's coefficient, and dimensional ratios for real fastener techniques and predict fatigue crack growth life for cold-worked holes [12] or to assist in design of structures subjected to pitting corrosion phenomenon [13]. The theoretical solutions presented also permit to relate the cold-working pressure to both the size of plastically deformed region and the size of the permanent radial expansion which are used in such problems as riveted holes or applications with a remote load [10]. Simple engineering displacement solutions such as those obtained in the present study are also required for an aeronautic industry in prolonging the life of structures by interference fit or coldworking technique [14].

5.1. General Conclusions. On the basis of rigorous analytical procedure developed for radial displacements, two simplified engineering formulae for steel structural applications are proposed and carefully analyzed. The first approximate formula (25) may be safely used in terms of numerical accuracy for bolted connection where the plastic zone surrounding a fastener is comparable in size with the outer elastic zone. In contrast, the second approximate formula (26) may be only used when plastic zones are extended, that is, in such applications as cold expansion of holes. The results obtained by this formula are on the safe side and differ from exact solution within $4 \%$ for real values of Poisson's ratio $(\nu=0.3)$. The advantage of the second approximate solution (26) is in its simplicity and physical consistency.

In general, for structural steel plate applications, consideration of elastic strains in the inner plastic zone leads to lower values of radial displacements especially for smaller values of Poisson's ratio, closer to the edge of the hole and upon higher external loading. It should be also reminded, however, that, in contrast to the displacement calculation procedures, the related strain analysis, that is, determination of strain fields, must be always conducted using rigorous formula (22) to avoid serious errors in accuracy or physical inconsistency and prevent accumulation of these errors in numerical codes.

\section{Conflict of Interests}

The author declares that there is no conflict of interests regarding the publication of this paper.

\section{References}

[1] T. N. Chakherlou and J. Vogwell, "The effect of cold expansion on improving the fatigue life of fastener holes," Engineering Failure Analysis, vol. 10, no. 1, pp. 13-24, 2003.

[2] P. Moze and D. Beg, "A complete study of bearing stress in single bolt connections," Journal of Constructional Steel Research, vol. 95, pp. 126-140, 2014.

[3] J. Chakrabarty, Theory of Plasticity, Elsevier, New York, NY, USA, 3rd edition, 2006.

[4] Y. C. Hsu and R. G. Forman, "Elastic-plastic analysis of an infinite sheet having a circular hole under pressure," Transactions ASME, Journal of Applied Mechanics, vol. 42, no. 2, pp. 347-352, 1975.

[5] W. Y. Lu and Y. C. Hsu, "Elastic-plastic analysis of a flat ring subject to internal pressure," Acta Mechanica, vol. 27, no. 1-4, pp. 155-172, 1977.

[6] D. Durban, "An exact solution for the internally pressurized, elastoplastic, strain-hardening, annular plate," Acta Mechanica, vol. 66, pp. 111-128, 1987.

[7] P. R. Arora and K. R. Y. Simha, "Analytical and experimental evaluation of coldworking process for strain hardening materials," Engineering Fracture Mechanics, vol. 53, no. 3, pp. 371-385, 1996.

[8] W. R. Broughton, L. E. Crocker, and M. R. L. Gower, "Design requirements for bonded and bolted composite structures," NPL Report MATC (A) 65, 2002. 
[9] R. K. L. Su and W. H. Siu, "Nonlinear response of bolt groups under in-plane loading," Engineering Structures, vol. 29, no. 4, pp. 626-634, 2007.

[10] S. T. Pinho, H. B. Martins, P. P. Camanho, M. H. Santare, and P. M. S. T. de Castro, "Residual stress field and reduction of stress intensity factors in cold-worked holes," Theoretical and Applied Fracture Mechanics, vol. 44, no. 2, pp. 168-177, 2005.

[11] M. Guowei, S. Iwasaki, Y. Miyamoto, and H. Deto, "Plastic limit analyses of circular plates with respect to unified yield criterion," International Journal of Mechanical Sciences, vol. 40, no. 10, pp. 963-976, 1998.

[12] D. L. Ball, "Elastic-plastic stress analysis of cold expanded fastener holes," Fatigue and Fracture of Engineering Materials and Structures, vol. 18, no. 1, pp. 47-63, 1995.

[13] J. Nagler, "Pitting corrosion investigation of cantilever beams using F. E. method," World Journal of Mechanics, vol. 3, no. 2, pp. $89-100,2013$.

[14] G. Wanlin, "Elastic-plastic analysis of a finite sheet with a coldworked hole," Engineering Fracture Mechanics, vol. 46, no. 3, pp. 465-472, 1993. 

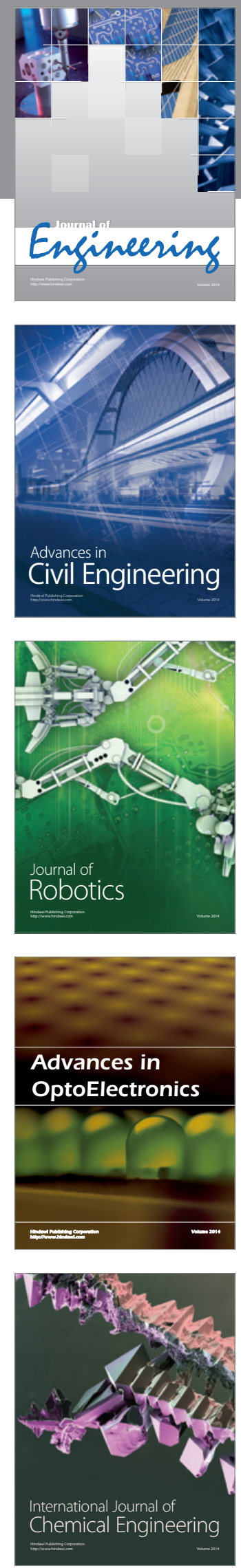

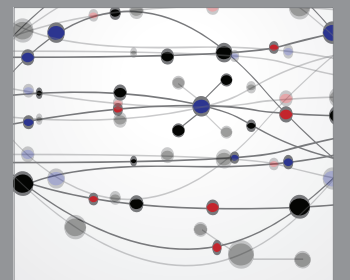

The Scientific World Journal
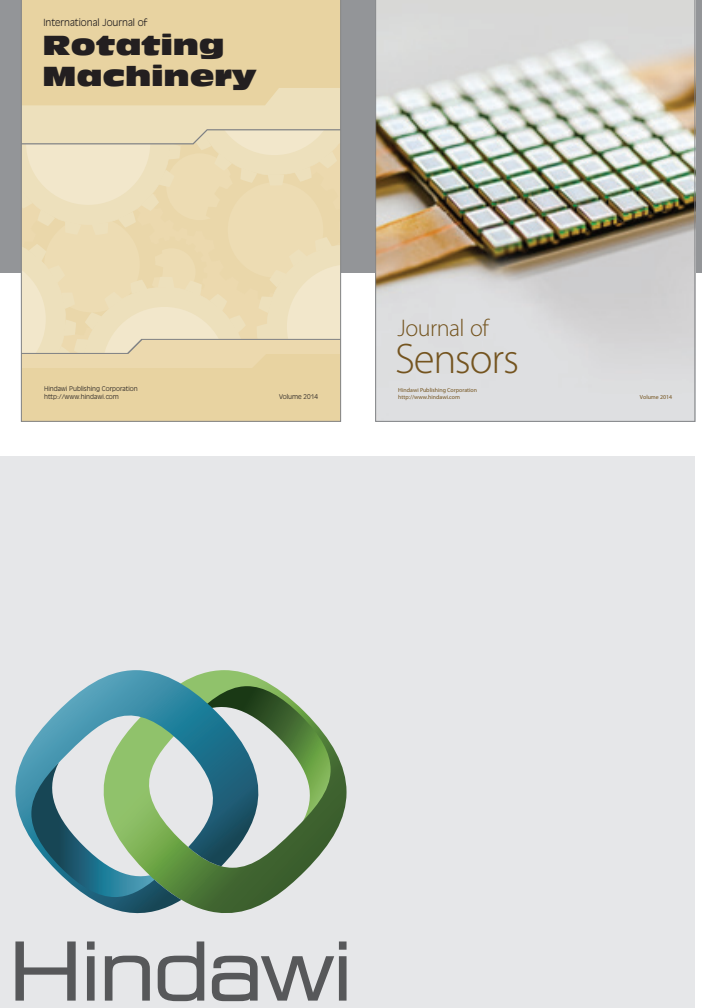

Submit your manuscripts at http://www.hindawi.com
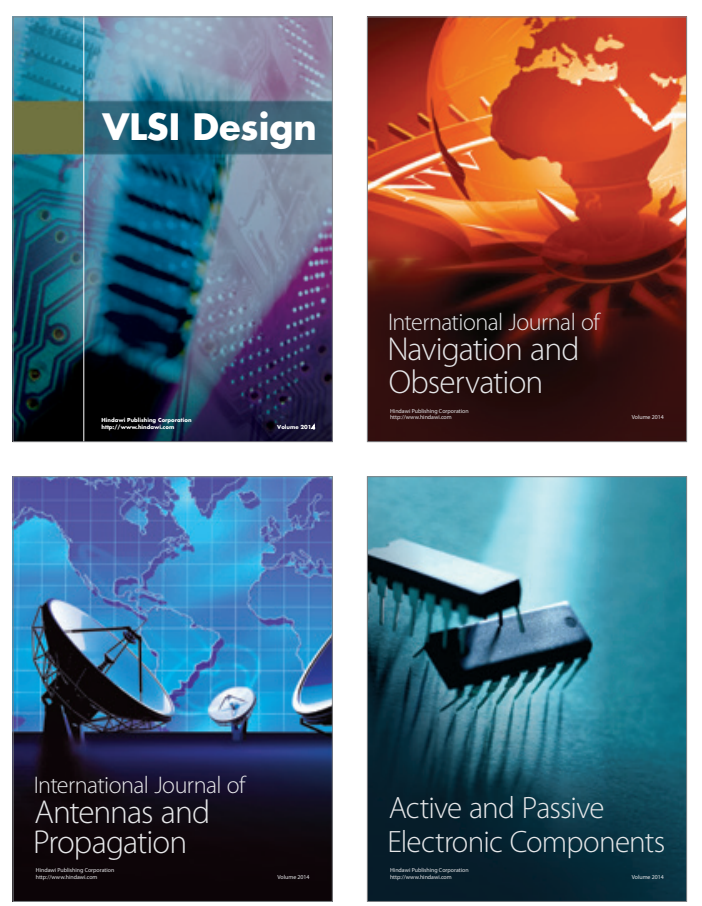
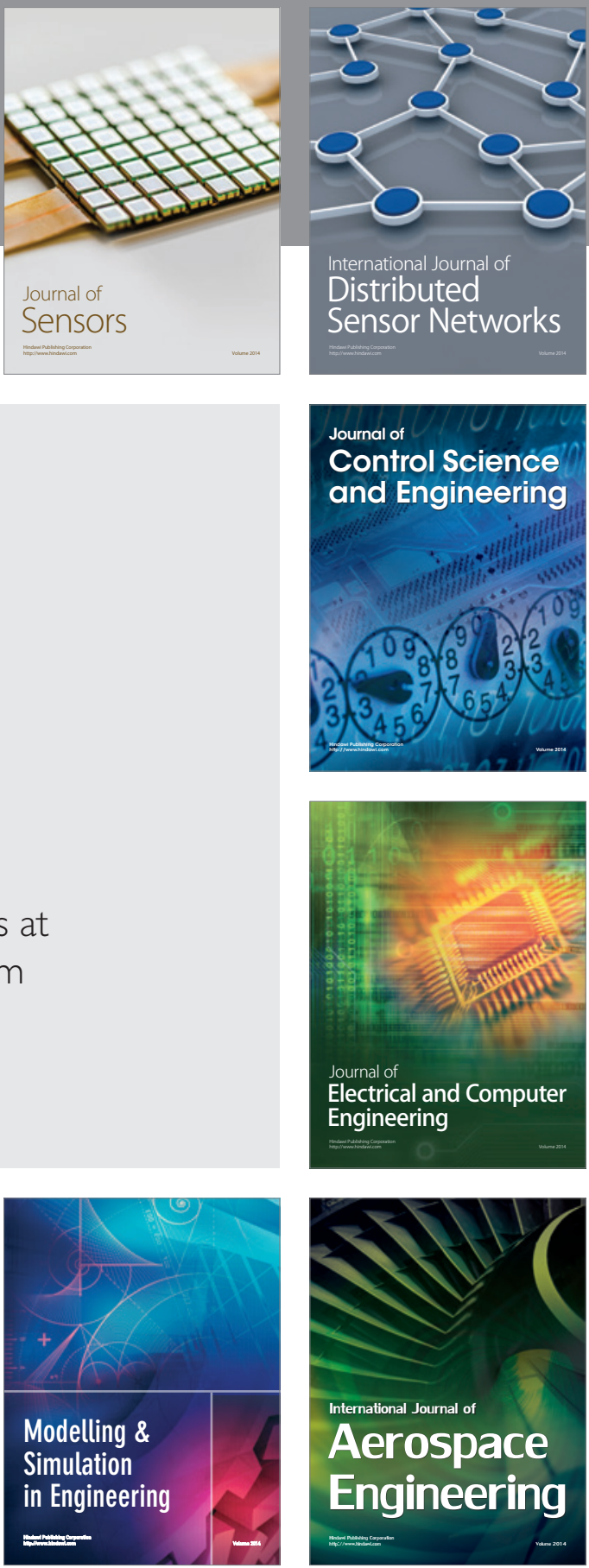

Journal of

Control Science

and Engineering
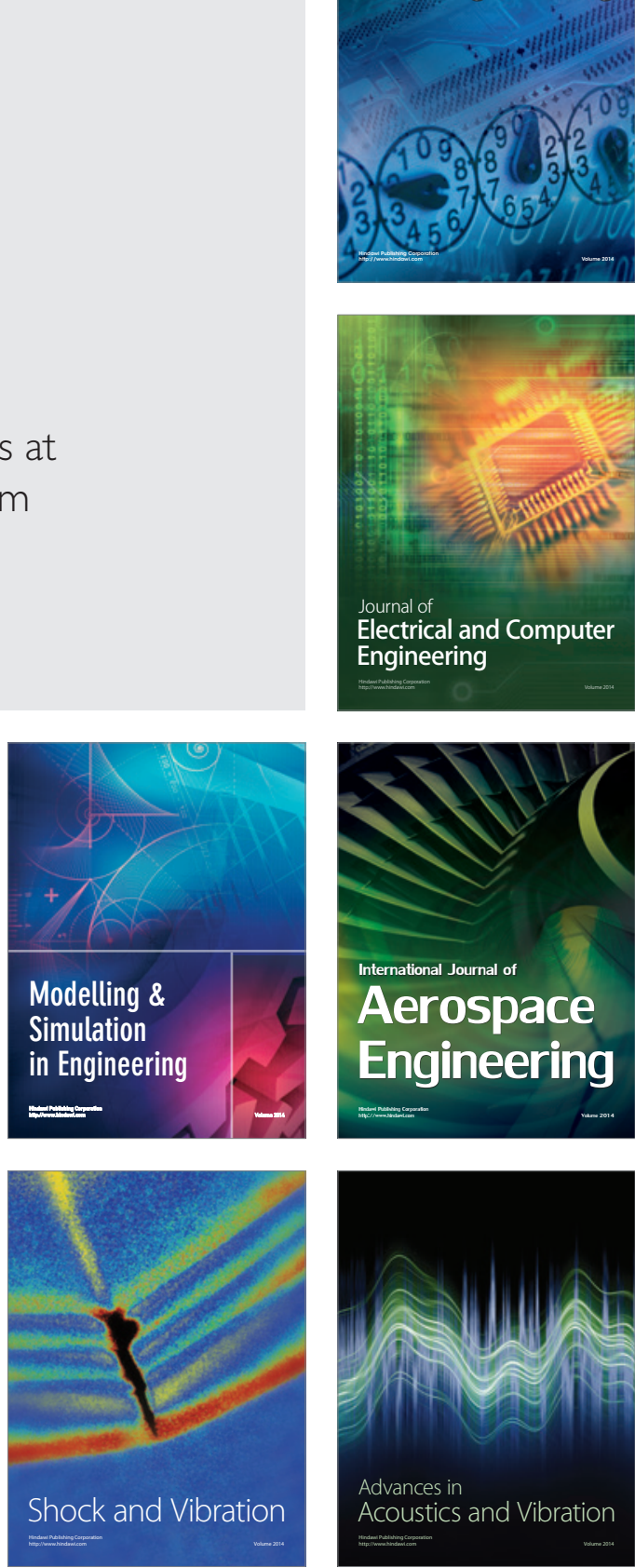